\title{
Recombinant Expression of Pleurocidin cDNA Using the Pichia pastoris Expression System
}

\author{
Olive-Jean Burrowes, ${ }^{1}$ Gill Diamond, ${ }^{2}$ and Tung-Ching Lee ${ }^{1}$ \\ ${ }^{1}$ Department of Food Science, Center for Advanced Food Technology, and Institute of Coastal \& Marine Sciences, \\ Rutgers, The State University of New Jersey, New Brunswick, NJ 08901, USA \\ ${ }^{2}$ Department of Oral Biology, New Jersey Dental School, \\ University of Medicine \& Dentistry of New Jersey (UMDNJ), 110 Bergen Street, Newark, NJ 07103-2495, USA
}

Received 27 September 2004; revised 13 April 2005; accepted 26 April 2005

\begin{abstract}
This research utilized the Pichia pastoris expression system for recombinant expression of cDNA of pleurocidin, a small (2.7 kd) antimicrobial peptide isolated from winter flounder (Pleuronectes americanus). The Pichia vector contains the alcohol oxidase gene promoter (AOX 1), which under the induction of methanol allows for expression of heterologous protein gene inserted downstream in the vector. Two strains of $P$ pastoris were used as host cells, the wild type $\left(P\right.$ pastoris $\left.\mathrm{X}-33^{\left(\mathrm{mut}^{+}\right)}\right)$and the mutant $(P$ pasatoris $\left.\mathrm{KM} 71 \mathrm{H}^{\left(\mathrm{mut}^{s}\right)}\right)$. Polymerase chain reaction (PCR) and DNA sequencing showed that pleurocidin cDNA was successfully integrated into the $P$ pastoris genome. Reverse transcription (RT)-PCR showed that pleurocidin was transcribed by both Pichia host strains. Affinity chromatography, SDS-PAGE, and immunological techniques were used for purification and detection of recombinant peptide. Although there was strong evidence of transcription of pleurocidin cDNA, the Pichia system requires further optimization to obtain detectable levels of this small peptide.
\end{abstract}

\section{INTRODUCTION}

Pleurocidin is a 25 -amino-acid peptide isolated from the skin mucus secretions of the winter flounder (Pleuronectes americanus) (Cole et al [1]). Pleurocidin exhibits broad-spectrum antimicrobial activity, is salt- and heattolerant, and is also noncytotoxic and nonhemolytic to human cells in vitro (Cole et al [1]; Burrowes et al [2]).

Pleurocidin has been found to be effective against clinical isolates from patients with urinary and respiratory tract infections and hip and bone wounds, and acts synergistically with $\mathrm{D}$-cycloserine against the food-related and medically significant Mycobacterium tuberculosis (Cole et al [3]). The amide structure of pleurocidin was found to reduce the mortality of coho salmon infected with Vibrio anguillarum and to be more effective against some fish pathogens than gramicidin S and polymyxin B (Jia

Correspondence and reprint requests to Tung-Ching Lee, Department of Food Science, Center for Advanced Food Technology, and Institute of Coastal and Marine Sciences, Rutgers, The State University of New Jersey, New Brunswick, NJ 08901, USA, E-mail: lee@aesop.rutgers.edu

This is an open access article distributed under the Creative Commons Attribution License which permits unrestricted use, distribution, and reproduction in any medium, provided the original work is properly cited. et al [4]). These findings suggest that pleurocidin has potential application in aquaculture, as well as for food and pharmaceutical applications.

The high cost of chemically synthesizing pleurocidin or isolating it directly from the winter flounder is prohibitive for the large-scale applications. However, producing the peptide in a microbiological system through the use of a genetically modified organism would allow for large-scale production and purification.

Pichia pastoris is a methylotropic yeast capable of utilizing methanol as a sole carbon source. The AOX 1 promoter, which regulates the AOX 1 gene allows for the production of the alcohol oxidase enzyme required for the oxidation of methanol and also allows for the overexpression of heterologous protein genes introduced downstream in a Pichia expression vector. This allows for the production of 10-100 times more recombinant protein than does the traditionally utilized Saccharomyces cerevisiae expression system (Higgins and Cregg [5]). Methanol induced $P$ pastoris cultures grown in shake-flask results in expression levels of approximately $5 \%$ of total soluble proteins. However, this level is increased to $>30 \%$ of total proteins in cells grown in fermenter cultures utilizing growthlimiting rates of methanol. A key factor is the preferential use of the $P$ pastoris system for respiratory growth, which facilitates its culturing at high-cell densities relative to fermentation yeasts such as Saccharomyces cerevisiae (Higgins and Cregg [5]; d'Anjou and Daugulis [6]; Villate et 
al [7]). The $P$ pastoris expression system allows for the recombinant protein to either be expressed intracellularly or to be secreted into the growth medium. Secretion is said to be the first step in purification of heterologous proteins produced from the Pichia system because it separates the recombinant protein from the bulk of the cellular proteins which are present in low concentrations in Pichia cells (Eldin et al [8]; Higgins and Cregg [5]; Shen et al [9]). Hence, the recombinant protein will form a major portion of the total protein in the expression medium.

The Pichia vectors carry the secretion signal sequence from the Saccharomyces cerevisiae $\alpha$-factor prepropeptide located downstream from the $A O X 1$ promoter and upstream from the heterologous protein gene, thus facilitating secretion of the expressed protein.

The vector used in our studies is the $\mathrm{pPICZ} \alpha$ vector designed by Invitrogen Corporation (Invitrogen Corporation, Calif). This vector contains functional sites which are designed for ease of integration of foreign protein genes, selection of transformants, purification and identification of the recombinant protein.

Several investigators including Eldin et al [8] and Baumgartner et al [10] have utilized the convenience of the secretion signal and hexahistidine tag contained in the $P$ pastoris vectors to express and purify recombinant proteins in a one-step purification procedure using affinity metal-chelating chromatography. Eldin et al [8] purified two single-chain antibody fragments, which were expressed at $250 \mathrm{mg} / \mathrm{L}$ and $60 \mathrm{mg} / \mathrm{L}$, respectively. Baumgartner's group expressed and purified the kidney bean lectin phytohemagglutinin E-form, which was secreted in the expression medium to levels of $16 \mathrm{mg} / \mathrm{ml}$. The use of both the wild-type methanol-utilizing strain $P$ pastoris $\mathrm{X}$ $33\left(\mathrm{mut}^{+}\right)$and the slow methanol-utilizing strain $P$ pastoris $\mathrm{KM} 71 \mathrm{H}\left(\right.$ mut $\left.^{-}\right)$to express various heterologous proteins is well documented (Bellevik et al [11]; Ogawa et al [12]; Reddy and Dahms [13]; Baumgartner et al [10]; and Feng et al [14]).

The objective of this research is to investigate the suitability of the $P$ pastoris expression system as a means of recombinant expression of pleurocidin. The rationale for the use of this expression system in our research is based on the previously outlined features of the $P$ pastoris expression system as well as the widely documented success of previous workers utilizing this system. Other expression systems such as the the Escherichia coli expression system was not used in our research because pleurocidin is being investigated as a potential food preservative, $E$ coli is perceived as being a dangerous pathogen, therefore using E coli would not have been appropriate for the expression of pleurocidin from a consumer acceptance perspective, and therefore using $E$ coli would not achieve our objective. However, $P$ pastoris is ideally suited as a means of producing pleurocidin for human use, because Pichia cells have been previously used as a source of animal feed by the Phillips Petroleum Company, and would therefore be much more acceptable from a consumer standpoint.
Moreover, expression in yeast would allow for the ease of upscaling from benchtop to large fermentation volumes that would be required if pleurocidin is to be produced in sufficiently large quantities for future commercial use.

It is noted, however, that the majority of reported studies using the Pichia system have been with relatively large molecules $(20-80 \mathrm{kd})$. A major challenge in our studies is that we aim to express a small peptide (pleurocidin), which is, indeed, a very small peptide $(2.7 \mathrm{kd})$.

\section{MATERIALS AND METHODS}

\section{Vector selection and cloning}

The EasySelect Pichia Expression System was used for these genetic engineering studies. All primers, vectors, and host cells were obtained from Invitrogen Corporation (Invitrogen Corporation, Calif). The vector $(\mathrm{pPICZ} \alpha)$, pleurocidin cDNA (obtained from Dr. Gill DiamondUMDNJ), and primers were forwarded to Commonwealth Biotechnologies Inc (Richmond, Va), where in collaboration with our laboratory, the pleurocicin cDNA was cloned into the pPICZ $\alpha$ vectors and returned to our laboratory for transformation and expression experiments.

Two clones were constructed, a native 75 base-pair pleurocidin fragment $(\mathrm{pPICZ} \alpha \mathrm{A})$ and a modified 75 base-pair fragment in which several nucleotides were changed to match the codon sequences preferentially used by $P$ pastoris cell ( $\mathrm{pPICZ} \alpha \mathrm{B})$. The changes made in pPICZ $\alpha$ B were as follows.

(i) Alanine (19) codon changed from GCG to GCT (GCG tRNA is rare in Pichia).

(ii) The segment sequenced phenylalanine ( $5 \& 6$ ) and lysine (7 \& 8) having codons TTT TTT AAA AAA were modified to TTC TTC AAG AAG as AT rich regions have been demonstrated to prematurely terminate transcription in Pichia.

(iii) The terminal codons TAC and CTT for tyrosine and leucine, respectively, were changed to read TAT and CCT to facilitate direct fusion of the pleurocidin cDNA to the myc-epitope.

\section{Transformation of P pastoris host strains}

Two strains of $P$ pastoris commonly used in heterologus protein expressions were selected for transformation with the pleurocidin cDNA in our laboratory; Pichia host cells $P$ pastoris $\mathrm{X}-33^{\left(\mathrm{mut}^{+}\right)}$, a wild type Pichia strain containing the $A O X 1$ promoter which allows for rapid growth while utilizing methanol as the sole carbon source; and $\mathrm{KM} 71 \mathrm{H}^{\left(\mathrm{mut}^{\mathrm{s}}\right)}$, a mutant strain containing a disrupted $A O X 1$ promoter gene resulting in a slow methanolutilizing strain. Both cultures were made competent and transformed in our laboratory by introduction of the linearized pPICZ $\alpha$ vectors into these cells using the EasySelect Pichia Expression System. (Invitrogen Corporation, Calif). Transformed cells were selected by growth on yeast extract peptone dextrose (YPD) agar plates containing zeocin $(100 \mu \mathrm{g} / \mathrm{mL})$. 


\section{Preparation of competent cells}

Pichia host cells were grown in YPD broth overnight $\left(30^{\circ} \mathrm{C} / 250 \mathrm{RPM}\right)$ and prepared for transformation with the pPICZ $\alpha$ vector containing the pleurocidin insert, according to the manufacturer's recommendations (Invitrogen Corporation, Calif). Competent cells were stored at $-80^{\circ} \mathrm{C}$ until the time of transformation.

\section{Transformation by polyethylene glycol (PEG) method}

The linearized vector containing the pleurocidin cDNA was added to competent cells and transformation of $P$ pastoris host cells ( $P$ pastoris $\mathrm{X}-33^{\left(\mathrm{mut}^{+}\right)}$and $\mathrm{KM}_{71} \mathrm{H}^{\left(\mathrm{mut}^{\mathrm{s}}\right)}$ ) was performed using the PEG method according to the EasySelect Pichia instruction manual (Invitrogen Corporation, Calif). Transformants were selected on zeocin-containing medium (YPDS $+100 \mu \mathrm{g} / \mathrm{mL}$ zeocin).

\section{Determination of methanol-utilizing (mut) phenotype}

In order to determine the length of time required for expression of recombinant protein by the transformed $P$ pastoris host cells, it is necessary to determine the methanol utilization (mut) phenotype of the strain. The mut phenotype for the transformed Pichia X-33 strain was determined by the procedure outlined in Invitrogen's Easy Select Pichia expression manual. Briefly, growth of cells on minimal media with dextrose (MDH) was compared to growth on minimal media using methanol $(\mathrm{MMH})$ in place of dextrose. The intensity of growth of test cells was then compared to growth of control strains Pichia GS115/HSA $\left(\right.$ mut $\left.^{s}\right)$ which produces albumin $(65 \mathrm{kd})$ while slowly metabolizing methanol as sole carbon source and GS115/LacZ $\left(\right.$ mut $\left.^{+}\right)$which produces $\beta$-galactosidase $(120 \mathrm{kd})$ while rapidly metabolizing methanol as sole carbon source. $\left(\right.$ mut $\left.^{+}\right)$cells grow normally on both media while $\left(\right.$ mut $\left.^{s}\right)$ cells grow normally on minimal media with dextrose but show negligible growth on minimal media with methanol.

\section{Expression experiments \\ $X-33$ expression}

Four isolated transformed colonies from each host strain were tested for expression of recombinant pleurocidin. Expression experiments were conducted according to the methods used by Feng et al [14] and Yan et al [15], based on the procedure outlined in Invitrogen's Easy Select Pichia expression manual, with some modifications.

Aliquots of expression medium were removed (time points $0,6,12,24,36,48,60,72,84,96$ hours) for $P$ pastoris $\mathrm{X}-33$ strain and $(0,24,48,72,96,120,144$, 168 hours) for the $P$ pastoris KM $71 \mathrm{H}$ strain. Simultaneously, 2 colonies of $P$ pastoris GS115/LacZ were used as (mut $^{+}$) intracellular expression control and 2 colonies of GS115/HSA were used as $\left(\right.$ mut $\left.^{s}\right)$ secretion control. Cell pellet and supernatant were separated and frozen in liquid nitrogen and stored at $-70^{\circ} \mathrm{C}$ for analysis by SDS polyacrylamide gel electrophoresis (PAGE) and Western blot.

\section{Preparation of cell pellets}

Pelleted, transformed cells were prepared for analysis by mechanically disrupting the cell wall using glass beads ( 0.5 microns in diameter) with buffer containing the protease inhibitor phenylmethylsulfonyl fluoride (PMSF), sodium phosphate buffer ( $\mathrm{pH}$ 7.4), EDTA, and glycerol (breaking buffer); the breaking buffer was used to prevent protein degradation during cell lysis.

\section{Purification by affinity chromatography}

Protein analysis (Bradford method) was performed to determine protein concentration of the transformed and expression control samples. The purification was performed in batch mode using a 10-milliliter nickel-charged agarose affinity chromatography column (Invitrogen Corporation, Calif).

\section{SDS PAGE analyses}

Time-point samples of transformed cells were concentrated to approximately 30-50\% using a Speed-Vac centrifugal concentrator (Savant Instruments Inc, Farmingdale, NY, USA) and used for electrophoretic analysis and Western blotting. Both secretion (GS115/HSA) and intracellular (GS115/LacZ) control samples were also analyzed by SDS PAGE and Western blotting. Electrophoresis was performed using Bio-Rad's Mini Protean II Redi-Gel System (Bio-Rad Laboratories, Hercules, Calif). Duplicate gels were made for electrophoretic analysis of each sample. One gel was stained for visualization of protein bands and the other used for Western blot analysis.

\section{WESTERN BLOTTING}

\section{(a) Anti-myc-antibody}

After separation by SDS-PAGE, protein bands were transferred to $0.2 \mu \mathrm{m}$ PVDF membrane and protein transfer allowed to proceed (100 volts for $1 \mathrm{~h}$ ) in $25 \mathrm{mM}$ Tris, $192 \mathrm{mM}$ glycine, 30\% methanol transfer buffer ( $\mathrm{pH} 8.3$ ). Transblotted PVDF membranes were probed with antimyc-alkaline phosphatase conjugate antibody, which recognizes the myc-epitope attached to the heterologus protein. Color development was performed using the alkaline phosphatase chromogenic substrate conjugate kit. (BioRad Laboratories, Calif).

\section{(b) Anti-pleurocidin antibody}

Concurrent with the use of anti-mycalkaline phosphatase conjugate antibody, transblotted PVDF membranes were probed with pleurocidin antisera. Synthetic pleurocidin was used as control for the pleurocidin antisera. Synthetic pleurocidin (Ple) showed a strong band on SDS PAGE gel at approximately $3000 \mathrm{kd}$ (Figure 7) and on Western blot using the anti-pleurocidin antisera 
(1:100 dilution) as primary antibody with anti-rabbit alkaline phosphatase conjugate (1:20,000 dilution) as secondary antibody (Figure 8). Lanes 1-4 contained induced-time course samples from each of 2 clones of $P$ pastoris $\mathrm{X}-33^{\left(\mathrm{mut}^{+}\right)}$and $P$ pastoris $\mathrm{KM} 71 \mathrm{H}^{\left(\mathrm{mut}^{\mathrm{s}}\right)}$, respectively.

\section{Two-step purification procedures}

Expression experiments were scaled up (from $1 \times 250 \mathrm{~mL}$ flasks to $4 \times 250 \mathrm{~mL}$ flasks) in order to provide greater cell mass for induction, and hence enhancing the possibility of obtaining detectable expression of heterologous protein. Induction was conducted using buffered methanol minimal medium (BMMH) instead of buffered methanol complex medium (BMMY).

$1 \%$ casamino acid was added to the medium to inhibit any possible activity of extra cellular proteases. The expression medium was subjected to dialysis using a $6-8 \mathrm{kd}$ cut-off dialysis membrane (Spectrum Medical Industries Inc, Calif). Dialyzed samples were then purified by affinity chromatography as previously outlined in this paper.

\section{Polymerase chain reaction (PCR) and sequencing}

PCR analysis was conducted on transformed cells of both Pichia strains, in order to confirm whether the pleurocidin cDNA was actually integrated into the $P$ genome. Four clones, two from each host strain ( $P$ pastoris $\mathrm{X}$ $33^{\left(\mathrm{mut}^{+}\right)}$and $P$ pastoris $\left.\mathrm{KM} 71 \mathrm{H}^{\left(\mathrm{mut}^{s}\right)}\right)$ were tested according to standard protocol for PCR analysis.

DNA was extracted from cells transformed with the vector carrying the pleurocidin cDNA, using the Easy DNA kit for genomic DNA isolation (Invitrogen Corporation, Calif). PCR products of the extracted DNA were then purified for sequencing using Qiagen's QIAquick purification kit (Qiagen, Valencia, Calif).

Purified PCR fragments were sequenced (Biotechnology Center, Plant Science Department, Rutgers University) in order to ascertain orientation of inserted cDNA into the Pichia host cell genome, as well as to reveal whether there were any mutations in the inserted fragment.

\section{RNA extraction and reverse transcription}

Time-point expression samples of 4 clones, two each from strains $\left(P\right.$ pastoris $\mathrm{X}-33^{\left(\mathrm{mut}^{+}\right)}$and $P$ pastoris $\mathrm{KM}_{71} \mathrm{H}^{\left(\mathrm{mut}^{\mathrm{s}}\right)}$ ) were tested for transcription of the pleurocidin cDNA by the transformed host strains.

\section{RNA extraction}

In order to facilitate the release of RNA from the transformed yeast cells, the cells were subjected to mechanical disruption using a high-speed bead beater, RNA was extracted from disrupted cells according to the protocol of the Qiagen RNeasy extraction kit (Qiagen, Calif). All samples were subjected to on-column DNase digestion using the Qiagen RNase free DNase treatment (Qiagen, Cailf) during RNA isolation. Isolated RNA was used as template in reverse transcriptase reaction.

\section{RT-PCR analysis was conducted in two parts}

(a) CDNA synthesis

First strand cDNA synthesis was performed using the SuperScript II RT kit (Invitrogen Corporation, Calif). Extracted yeast $\mathrm{RNA}^{+}$reverse transcriptase enzyme using oligo (dT) primers was tested using RNA without reverse transcriptase enzyme as control. The cDNA produced from the RT reaction was then used as template for amplification by PCR.

\section{(b) Polymerase chain reacton (PCR)}

PCR reaction was performed as previously mentioned. PCR controls included PCR mix without added cDNA template (negative control), and pPICZa vector containing gene insert (positive control). Both induced and uninduced samples were analyzed.

\section{Agarose gel electrophoresis of PCR product}

Samples of PCR product were loaded onto 1\% agarose gel for electrophoretic separation (100 volts for $35 \mathrm{~min}$ ). Separated bands were visualized using ultraviolet light after staining in ethidium bromide $(1 \mu \mathrm{g} / \mathrm{mL})$ in Trisacetate-EDTA (TAE) buffer (30 min).

\section{Analysis of transcription samples}

Samples showing transcription of cDNA in RT-PCR analysis were purified by metal-chelating affinity column chromatography as previously outlined in this paper. $\mathrm{Pu}-$ rified fractions showing a relative increase in protein concentration were analyzed by SDS PAGE and Western blot. The samples used were either supernatant from expression medium, uncentrifuged expression medium (whole broth), or lysate from cell pellets of the expression medium.

\section{RESULTS AND DISCUSSION}

The methanol utilization (mut) phenotype of the Pichia host strain $P$ pastoris X-33 transformed with pleurocidin cDNA was confirmed to be mut ${ }^{+}$phenotype as shown in Figure 1. All $P$ pastoris KM71H strains are mut ${ }^{s}$ phenotype due to their disrupted $A O X 1$ Promoter, therefore it was not necessary to determine the mut phenotype for this strain.

Initial expression experiments with transformed $P$ pastoris cells showed that the cells used as expression control ( $P$ pastoris/GS115/LacZ ${ }^{\left(\text {mut }^{+}\right)}$) did indeed express the recombinant protein ( $\beta$-galactosidase) which was purified by the nickel-agarose affinity chromatography column selective for his-tag proteins. Expression of $\beta$-galactosidase was indicated by a protein band shown in the first fraction (F1) eluted from the affinity column anddetected on SDS 


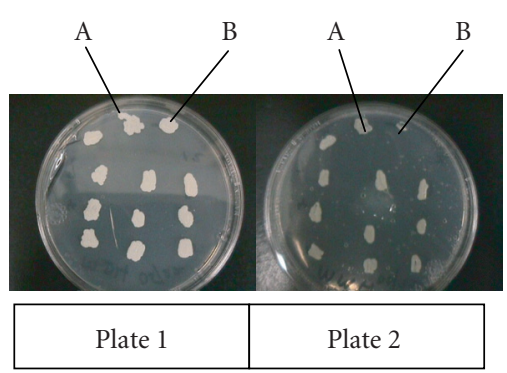

FIgURE 1. Methanol utilization (mut) phenotype of transformed Pichia pastoris X-33. A represents colony of $P$ pastoris control strain $P$ pastoris $/$ LacZ/ $\beta$-galactosidase $\left(\right.$ mut $\left.^{+}\right)$strain. B represents colony of $P$ pastoris/HSA/albumin $\left(\right.$ mut $\left.^{s}\right)$. Plate (1): minimal media with dextrose (MMD). Plate (2): minimal media with methanol (MMH).

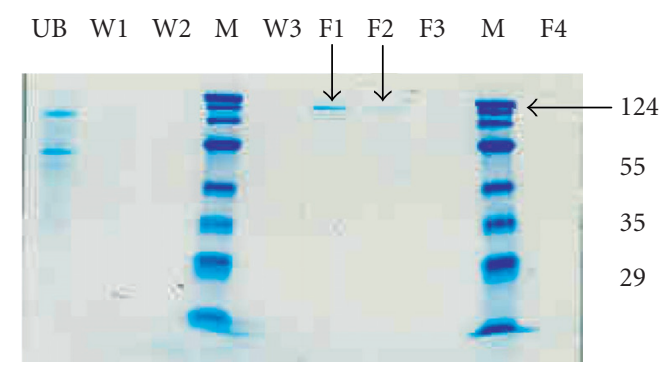

Figure 2. SDS PAGE gel of purified fractions of $\beta$-galactosidase expressed by $P$ pastoris $\mathrm{GS} 115 / \mathrm{LacZ} / \mathrm{mut}^{+}$expression control cells, eluted from affinity column. $M$ represents molecular weight marker; UB represents unbound fraction. W1-W3 represent wash fractions; F1-F4 represent eluted fractions. Purified recombinant $\beta$-galactosidase eluted in fraction 1 (F1) is indicated by band at approximate $124 \mathrm{kd}$.

PAGE at approximately $124 \mathrm{kd}$ (Figure 2). Western blot of the SDS PAGE gel showed the reaction of the myc-epitope attached to the recombinant $\beta$-galactosidase with the antimyc-antibody (Figure 3). The almost indistinguishable $\beta$ galactosidase band in fraction 2 (F2) on the SDS PAGE gel was clearly visible on the Western blot indicating the increased sensitivity of the Western blot analysis relative to the SDS PAGE.

A Western blot analysis representative of the results obtained from expression experiments of the $P$ pastoris cells transformed with the pleurocidin cDNA is shown in Figure 4 . The lack of visible bands in lanes 1-6 which represents time-course samples $(12,24,48,72,84,96 \mathrm{~h})$ from $P$ pastoris $\mathrm{X}-33^{\left(\mathrm{mut}^{+}\right)}$induction indicates that there was no detectable level of recombinant pleurocidin obtained in these expression experiments. The validity of these expression experiments was, however, confirmed by the expression of recombinant $\beta$-galactosidase by the $P$ pastoris GS115/LacZ/mut ${ }^{+}$control cells (Figure 4, lane 7 ) which was conducted simultaneously on the test cells.

The demonstration of the presence of expressed recombinant protein by the control samples indicates that

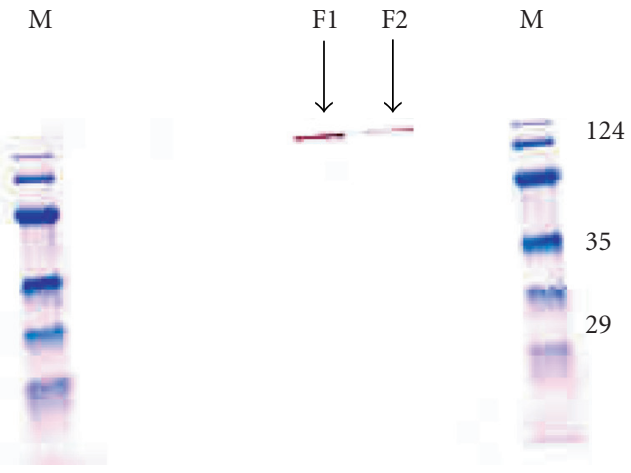

FIgURE 3. Western blot obtained from transfer of duplicate SDS PAGE gel containing purified fractions of recombinant $\beta$ galactosidase expressed by $P$ pastoris GS115/LacZ/mut ${ }^{+}$cells and showing reaction of the myc-epitope of the recombinant $\beta$ galactosidase with the anti-myc-antibody. Purified recombinant $\beta$-galactosidase eluted in fractions 1 and 2 (F1 and F2) are indicated by band at approximately $124 \mathrm{kd}$. (F2 band only faintly shown on SDS PAGE gel).

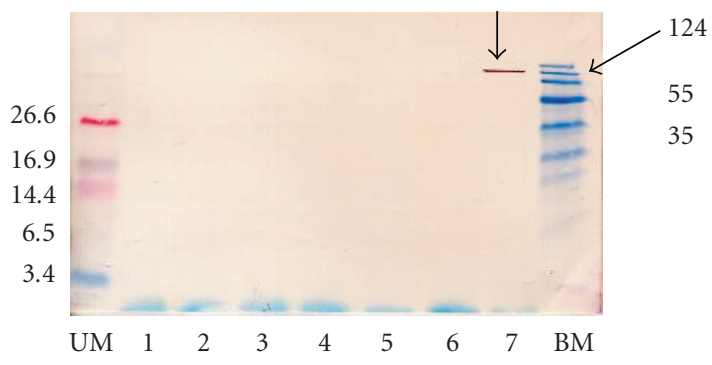

Figure 4. Western blot (using the anti-myc-antibody) for timecourse samples of induced transformed Pichia pastoris X-33 cells. UB represents ultra-low marker (peptides). BM represents broad-range marker (proteins). 1-6: $P$ pastoris X-33 time-course induced samples. 7: P pastoris LacZ expression control (48 h).

the procedures used for expression and purification are appropriate for $P$ pastoris transformation systems. However, there was no detection of protein bands on SDS gel electrophoresis or Western blot analysis for test samples. Similar results were obtained for the mutated $P$ pastoris $\mathrm{KM} 71 \mathrm{H}^{\left(\mathrm{mut}^{\mathrm{s}}\right)}$ strain.

In order to ascertain whether the absence of detectable expression of recombinant pleurocidin was due to lack of, or incorrect integration of the pleurocidin CDNA into the $P$ pastoris host cell genome, PCR was performed on the transformed yeast DNA followed by DNA sequencing. Agarose gel electrophoresis of the PCR product of transformed $P$ pastoris cells showed that the vector containing the cDNA of pleurocidin was indeed integrated in all four clones tested. This is indicated by strong bands at approximately $590 \mathrm{bp}$ in lanes $2-5$ of the agarose gel of the PCR product (Figure 5). These lanes represent transformed samples of both strains of $P$ pastoris used in this study. Lanes 6-7 consist of samples from untransformed 


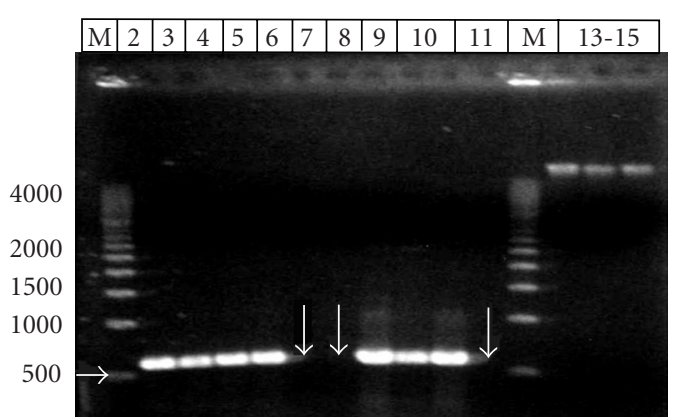

FIgURE 5. PCR analysis of transformed Pichia pastoris cells showing inserted pleurocidin cDNA at $590 \mathrm{bp}$. M represents 500 bp marker. Lanes $2-5$ represent transformed DNA ( $P$ pastoris $\mathrm{KM} 71 \mathrm{H}$ and $\mathrm{X}-33$ ). Lanes 6-7 represent control DNA (untransformed cells in lanes) indicated by parrallel arrows. Lanes 8-9 represent vector DNA (vector with and without insert). Lane 10 represents vector with transformed DNA. Lane 11 represents PCR control (no DNA) indicated by single arrow. Lanes 13-15 represent yeast DNA.

$P$ pastoris cells (indicated by parallel arrows). The absence of visible bands in untransformed cells and presence of the expected bands $(590 \mathrm{bp})$ in the transformed cells verifies the fact that pleurocidin cDNA has been inserted in the Pichia host cells. Lanes 8-10 consist of the linearized pPICZ $\alpha$ vector carrying the pleurocidin $\mathrm{cDNA}$ and the uncut $\mathrm{pPICZ} \alpha$ vector without pleurocidin cDNA. Lane 11 indicated by the single arrow consists of the PCR mix without extracted DNA (negative control). The absence of a DNA band in lane 11 verifies that there was no DNA contamination in the PCR samples. Yeast DNA observed in lanes 12-14 was included in the test to verify that DNA was indeed extracted from the yeast cells in the event there were no visible DNA bands from the transformed cells.

DNA sequencing of the purified PCR product confirmed that the pleurocidin gene sequence was correctly inserted into the yeast genome. The sequence obtained from the sequencing chromatogram file showed that the start of the alpha-factor sequence $\left(\mathrm{GAT}^{1}\right)$, the pleurocidin sequence, myc-epitope, and polyhistidine tag followed in the correct sequence (Figure 6), as verified by comparison with the sequence obtained by the designers and manufacturers of the vectors (Invitrogen Corporation, Calif) and (Commonwealth Laboratories, Richmond, Va).

Confirmation of the proper integration of the pleurocidin coding sequence into the Pichia genome encouraged further investigations. Subsequent experiments involved induction of colonies from clones confirmed by PCR and DNA sequencing to contain the pleurocicin gene sequence. In addition to using the anti-myc-antibody to test for the presence of recombinant pleurocidin by these clones, the more specific anti-pleurocidin antibody was introduced at this stage of our research. Synthetic pleurocidin (Ple) was used as control for the pleurocidin antisera and showed a strong band on SDS PAGE gel at approximately $3000 \mathrm{kd}$ (Figure 7) and on Western blot using the anti-pleurocidin antisera (1:100 dilution) as primary antibody with anti-rabbit alkaline phosphatase conjugate (1:20,000 dilution) as secondary antibody (Figure $8)$. Lanes 1-4 contained induced time-course samples from each of 2 clones of $P$ pastoris $\mathrm{X}-33^{\left(\mathrm{mut}^{+}\right)}$and $P$ pastoris $\mathrm{KM} 71 \mathrm{H}^{\left(\mathrm{mut}^{s}\right)}$. The absence of detectable bands on both SDS PAGE gel and Western blot further indicated that there was no detectable level of recombinant pleurocidin either using the anti-myc-antibody or the more specific pleurocidin antisera.

Having established the proper orientation of the cDNA into the Pichia yeast genome, it is evident that nonexpression of recombinant pleurocidin is not due to lack of modification of the Pichia cells, therefore in order to ascertain whether the lack of detection of expressed recombinant pleurocidin was due to a transcription related problem, or whether the gene is actually being transcribed, reverse transcriptase analysis was conducted using induced and uninduced transformed cells. The results of the RT-PCR analyses show that the pleurocidn gene is indeed being transcribed by the Pichia system in both the slow methanol-utilizing strain $\left(\mathrm{KM} 71 \mathrm{H}^{\mathrm{mut}}{ }^{\mathrm{s}}\right)$ and the wild-type methylotrophic strain (X-33 mut ${ }^{+}$) of transformed $P$ pastoris. Transcription of the pleurocidin cDNA was demonstrated by the presence of DNA bands (590 bp) in lanes 11 and 12 (indicated by arrows) on agarose gel from the RT-PCR products (Figure 9a). Lanes 11-12 represent induction samples collected on days 5 and 6 for the P pastoris KM71 ${ }^{\text {mut }^{s}}$ strain. Figure $9 \mathrm{~b}$ represents duplicates of the samples in Figure 9a, without the reverse transcriptase (RT) enzyme added (no RT control). The absence of visible bands in the no-RT controls verifies the authenticity of the bands observed in the presence of the RT enzyme in Figure 9a. Transcription of the pleurocidin cDNA on day 3 for two different clones of $\left(\mathrm{X}-33 \mathrm{mut}^{+}\right)$was indicated by DNA bands in lanes 21 and 25 indicated by arrows on agarose gels shown in Figure 10a. Lanes 21 and 25 contain induced samples collected on day 3 for the two clones. Figure $10 \mathrm{~b}$ represents the no-RT controls.

The greater intensity of the band observed with the $P$ pastoris $\left(\mathrm{KM} 71^{\mathrm{mut}}{ }^{s}\right)$ compared to the band observed for the $P$ pastoris $\left(\mathrm{X}-33^{\mathrm{mut}^{+}}\right.$) strain indicates that there might be a greater level of expression by the $\left(\mathrm{KM} 71^{\mathrm{mut}^{\mathrm{s}}}\right)$ strain, however, the appearance of the band shown at day 3 for the $\left(\mathrm{X}-33^{\text {mut }^{+}}\right)$strains and day 5 for the $\left(\mathrm{KM} 71^{\mathrm{mut}^{\mathrm{s}}}\right)$ strain confirms that induction does occur much later, and hence more slowly in the $\left(\mathrm{KM} 71^{\mathrm{mut}^{\mathrm{s}}}\right)$ strain. Figures 11 and 12 show that the protein production for both strains of transformed $P$ pastoris is inversely proportional to growth, indicating that protein expression occurs in the latter part of the growth phase of the cells.

This phenomenon could be a possible explanation for the reports of studies conducted by other investigators who state that protein expression in $P$ pastoris expression systems is greatly increased when conducted in continuous fermenter cultures compared to when conducted in flask (batch) cultures (Koganesawa et al [16]; Reddy and 


\begin{abstract}
JB-01-TCL sequence exported from chromatogram file TTTTACGACAACTTGNAGAAGATCAAAAAACAACTAATTATTCGAAACGAT ${ }^{1}$ GAGATTTCC TTCAATTTTTACTGCTGTTTTATTCGCAGCATCCTCCGCATTAGCTGCTCCAGTCAACAC TACAACAGAAGATGAAACGGCACAAATTCNGGCTGAAGCTGTCATCGGTTACTCAGATTT AGAAGGGGATTTCGATGTTGCTGTTTTGNCATTTTCCAACAGCACAAATAACGGG TCGAGAAAGAGAGGCTGAAGCTGCA GGC ${ }^{2}$ TGGGGAAGCTTCTTCAAGAAGGCTGCTCACGT TGGCAAGCATGTTGGCAAGGCTGCCCTTACTCATTATCTA GAA ${ }^{3}$ CAAAAACTCATCTCAGA AGAGGATCTGAATAGCGCCGTCGACCAT ${ }^{4}$ CATCATCATCATCAT TGA ${ }^{5}$ GTTTGTAGCCTTAG ACATGACTGTTCCTCAGTTCAAGTTGGGCACTTACGAGAAGACCGGTCTTGCTAGATTCT AATCAAGAGGATGTCAGAGTGCCATTTGCA

JB-02-TCL sequence exported from chromatogram file TTTTACGACAACTTGAGAAGATCAAAAAACAACTAATTATTCGAAACGAT ${ }^{1}$ GAGATTTCCT TCAATTTTTACTGCTGTTTTATTCGCAGCATCCTCCGCATTAGCTGCTCCAGTCAACACT ACAACAGAAGATGAAACGGCNCAAATTCNGGCTGAAGCTGTCATCGGTTACTCAGATTTA GAAGGGGATTTCGATGTTGCTGTTTTGNCATTTTCCAACTCTCTCGAGAAAGAGAGGCTGAAG CTGCAGGC ${ }^{2}$ TGGGGAAGCTTCTTCAAGAAGGCTGCTCACGTTGGCAAGCATGTTGGCAAGGCT GCCCTTACTCATTATCTA GAA ${ }^{3}$ CAAAAACTCATCTCAGAAGAGGATCTGAATAGCGCCGTCGA CCAT 4CATCATCATCATCATTGA ${ }^{5}$ GTTTGTAGCCTTAGACATGACTGTTCCTCAGTTCAAGTTGG GCACTTACGAGAAGACCGGTCTTGCTAGATTCTAATCAAGAGGATGTCAGAATGCCATTTGCA

JB-03-TCL sequence exported from chromatogram file TTNTACGACAACTTGAGAAGATCAAAAAACAACTAATTATTCGAAACGAT ${ }^{1}$ GAGATTTCCT TCAATTTTACTGCTGTTTTATTCGCAGCATCCTCCGCATTAGCTGCTCCAGTCAACACT ACAACAGAAGATGAAACGGCACAAATTCCGGCTGAAGCTGTCATCGGTTACTCAGATTTA GAAGGGGATTTCAATCTCTCGAGAAAGAGAGGCTGAAGCTGCA GGC ${ }^{2}$ TGGGGAAGCTTCTTCA AGAAGGCTGCTCACGTTGGCAAGCATGTTGGCAAGGCTGCCCTTACTCATTATCTA GAA ${ }^{3}$ CAA AAACTCATCTCAGAAGAGGATCTGAATAGCGCCGTCGACCAT ${ }^{4}$ CATCATCATCATCATTGA $^{5}$ GT TTGTAGCCTTAGACATGACTGTTCCTCAGTTCAAGTTGGGCACTTACGAGAAGACCGGTCTTG CTAGATTCTAATCAAGAGGATGTCAGAGTGCCATTTGCA

JB-04-TCL sequence exported from chromatogram file TTTTACGCAACTTGAGAAGATCAAAAAANAACTAATTATTCGAAANGAT ${ }^{1}$ GAGATTTCCTT CAATTTTTACTGCTGTTTTATTCGCAGCATCCTCCGCATTAGCTGCTCCAGTCAACACTA CAACAGAAGATGAAACGGCACNAATTCCGGCTGAAGCTGTCATCGGTTACTCAGATTTAG AAgGGGATTTCAACTCTCGAGAAAAGAGAgGCTGAAGCTGCA GGC ${ }^{2}$ TGGGGAAGCTTCTTCAA GAAGGCTGCTCACGTTGGCAAGCATGTTGGCAAGGCTGCCCTTACTCATTATCTA GAA ${ }^{3}$ CAAA AACTCATCTCAGAAGAGGATCTGAATAGCGCCGTCGACCAT ${ }^{4}$ CATCATCATCATCATTGA $^{5}$ GTT TGTAGCCTTAGACATGACTGTTCCTCAGTTCAAGTTGGGCACTTACGAGAAGACCGGTCTTGC TAGATTCTAATCAAGAGGATGTCAGANTGCCATTTGCA
\end{abstract}

FIGURE 6. cDNA in ORF of yeast genome for all four clones. GAT ${ }^{1}$ start of alpha-factor sequence. GGC ${ }^{2}$ start of pleurocidin sequence. $\mathrm{GAA}^{3}$ start of myc-epitope. CAT ${ }^{4}$ Start of polyhistidine tag. TGA ${ }^{5}$ stop codon.

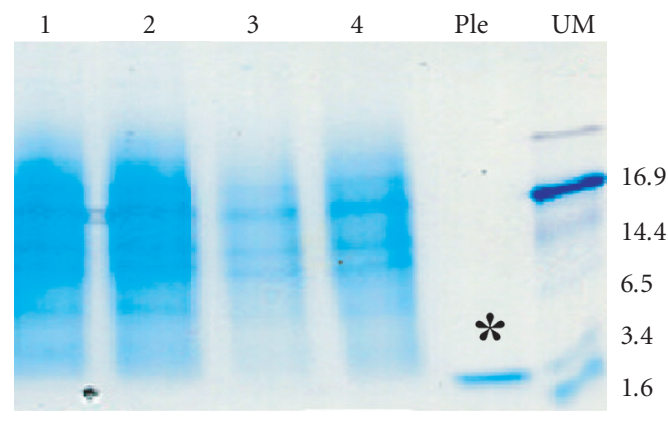

FIGURE 7. SDS polyacrylamide gel showing synthetic pleurocidin (Ple) band at appx $3000 \mathrm{kd}$.

Dahms [13]). In continuous fermentation systems, cell growth can be easily monitored, thus allowing growth to be maintained at the required stage for optimum production of protein. On the other hand, in flask cultures where growth is limited by the level of available nutrients, cells eventually enter the decline/death phase, and therefore

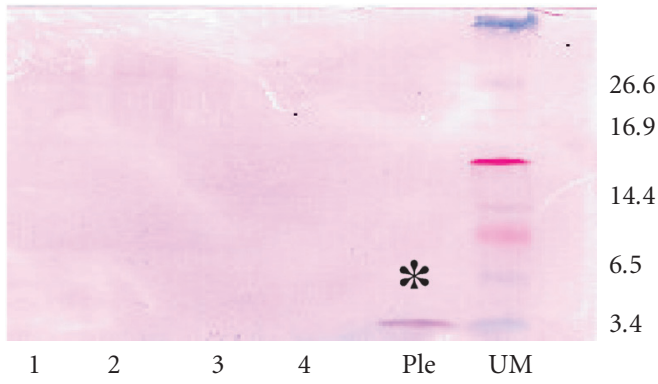

Figure 8 . Western blot of synthetic pleurocidin (Ple) using antipleurocidin antibody. 1-2: induced expresision samples of Pichia pastoris X-33 (days 2 and 4). 3-4: induced expresision samples of $P$ pastoris $\mathrm{KM} 71 \mathrm{H}$ (days 4 and 6). $\mathrm{UM}_{*}$ ultra-low marker (peptide marker). Ple: pleurocidin $(100 \mu \mathrm{g} / \mathrm{mL})$.

limit the amount of protein that can be obtained. Despite this possibility, several investigators have successfully expressed recombinant proteins in shake flasks, using the $P$ pastoris system (Villatte et al [7]; Paramasivam et al [17]; 


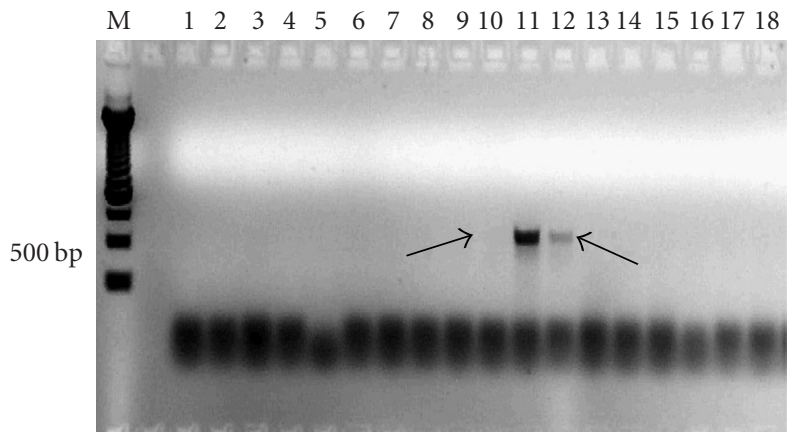

(a)

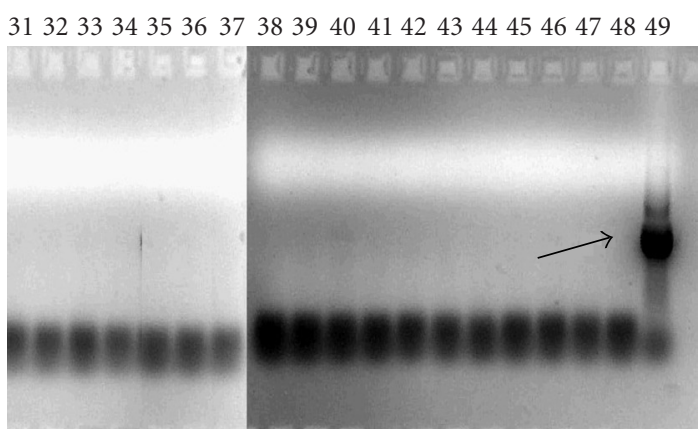

(b)

FIGURE 9. Transcribed pleurocidin cDNA bands at $590 \mathrm{~b}$ from transformed KMH 71cells at days 5 and 6 (+RT) and gel containing transformed KMH 71 cells (-RT); positive control shown in lane 48.

Li et al [18]). Thus, there are other factors to be considered for the lack of detection of recombinant pleurocidin.

The following set of induction experiments focused on utilizing those transformed cells showing transcription of the pleurocidin cDNA in the RT-PCR tests. Results of the isolation, purification, and detection procedures utilized for these transformed $P$ pastoris cells are shown in Figures $13 \mathrm{a}$ and $13 \mathrm{~b}$.

There was a strong band (indicated by arrows on each SDS PAGE gels) indicating the recombinant expression of $\beta$-galactosidase $(124 \mathrm{kd})$ on both SDS PAGE gel and Western blot, for the expression control cells ( $P$ pastoris GS115/LacZ), however, there was still an absence of detectable recombinant pleurocodin bands from transformed host cells in supernatant, intracellular fraction and purified whole broth fractions.

The evidence of expression of recombinant $\beta$ galactosidase by the $P$ pastoris GS115/LacZ ${ }^{\left(\text {mut }^{+}\right)}$expression control cells used throughout this research verifies the suitability of the methods and procedures used in this research.

Despite the absence of evidence of detectable expression of recombinant pleurocidin, our research has demonstrated that pleurocidin cDNA was successfully integrated into the pPICZ $\alpha$ vector and the $P$ pastoris system does allow for transcription of the pleurocidin cDNA, thus it is evident that RNA is indeed being produced and the lack of detection stems from a posttranscription problem.

Three main reasons are cited for the lack of expression of biologically active proteins in heterologous systems. These being host cell lacking components for translation of recombinant protein, the heterologous protein being prone to proteolysis as it emerges from translation, and incomplete or improper folding of heterologous protein (Butt RT, [19]). Although published information in the literature on the expression of small bioactive peptides like pleurocidin is very limited, it is well documented that a major limitation in the expression of small molecules (= $10 \mathrm{kd}$ ) is low or there is a lack of recombinant expression, often due to RNA instability (Rai and Padh [20]; Scorer et al [21]).

Additionally, studies reported by Sreekrishna et al [22] stated that two possible factors which influence protein expression in Pichia systems are (i) untranslated regions in recombinant mRNA and (ii) transcriptional and translational blocks. In our research, it is evident from the results of the RT-PCR experiments that mRNA is being transcribed (Figures 9 and 10). Thus it is the level of translation that is being affected. These results corroborates previous research which shows that the level of translation in small peptides is often a hinderance to recombinant expression of these small peptides. Pleurocidin being a small peptide consisting of only 25 amino acids is likely being degraded after translation.

The level of synthetic pleurocidin used as control in SDS PAGE electrophoresis and Western blotting was approximately $2.5 \mu \mathrm{g}(100 \mu \mathrm{g} / \mathrm{mL})$. However, SDS PAGE analysis of serially diluted synthetic pleurocidin samples indicate that as little as $325 \mathrm{ng}$ of pleurocidin can be detected by electrophoresis. It is evident therefore that if recombinant pleurocidin was indeed produced, it is below $325 \mathrm{ng} / \mathrm{mL}$.

This research is of significance because it is the first study reported on the utilization of a eukaryotic system $(P$ pastoris expression vectors) for the heterologous expression of the small novel antimicrobial peptide pleurocidin. Since in most cases it is impractical to express small peptides in the native host, continued research is required to modify existing systems to allow for optimal expression levels to be achieved.

Considering the advantages of the $P$ pastoris expression system over other recombinant expression systems, it would be beneficial to proceed with these optimization experiments, rather than change from the Pichia system to another expression system. We suggest that in the cloning for expression of small peptides like pleurocidin, optimization studies should involve the construction of $P$ pastoris vectors with multiple cloning sites to facilitate multiple copies of the gene to be integrated into the 


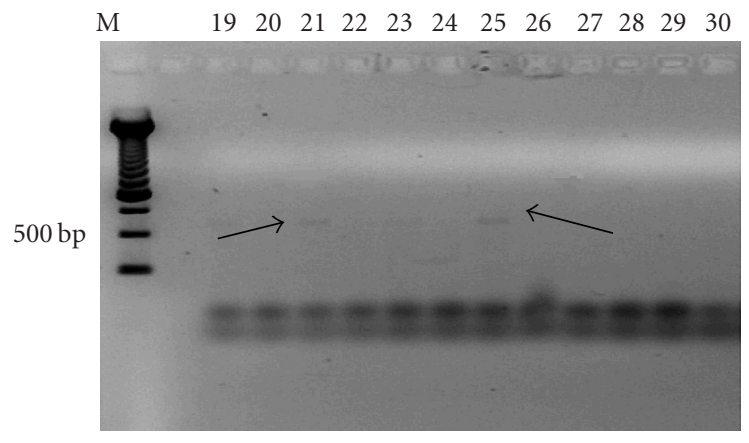

(a) $\begin{array}{llllllllllllll}49 & 50 & 51 & 52 & 53 & 54 & 55 & 56 & 57 & 58 & 59 & 60 & 61 & 62\end{array}$

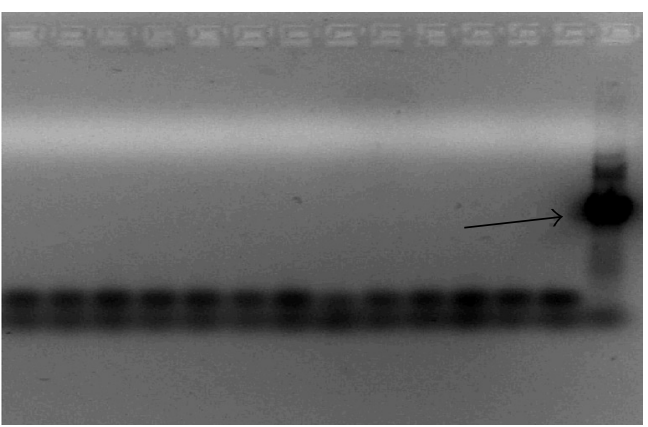

(b)

Figure 10. M: 250- bp DNA ladder. Lanes 1-6: time course samples of KM71H (clone 1) cells. Lanes 7-12: time course samples of $\mathrm{KM} 71 \mathrm{H}$ ( clone 2) cells. Lanes 13-18: time course samples of KM71H (uninduced) cells. Lanes 19-22: time course samples of X-33 (clone 1) cells. Lanes 23-26: time course samples of X-33 (clone 2) cells. Lanes 27-30: time course samples of X-33 (uninduced) cells. Lanes 31-47: duplicates of KM71H cells without reverse transcriptase enzyme (no RT control). Lanes 49-60: duplicates of X-33 cells without reverse transcriptase enzyme (no RT control). Lanes 48 \& 62 vector with pleurocidin DNA insert (positive control). Lane 61: PCR Master mix without DNA template (negative control). (a) Transcribed cDNA bands at 590 bp from transformed X-33 cells at day $3(+\mathrm{RT})$ and (b) gel containing transformed X-33 cells ( $-\mathrm{RT})$; positive control shown in lane 62 .

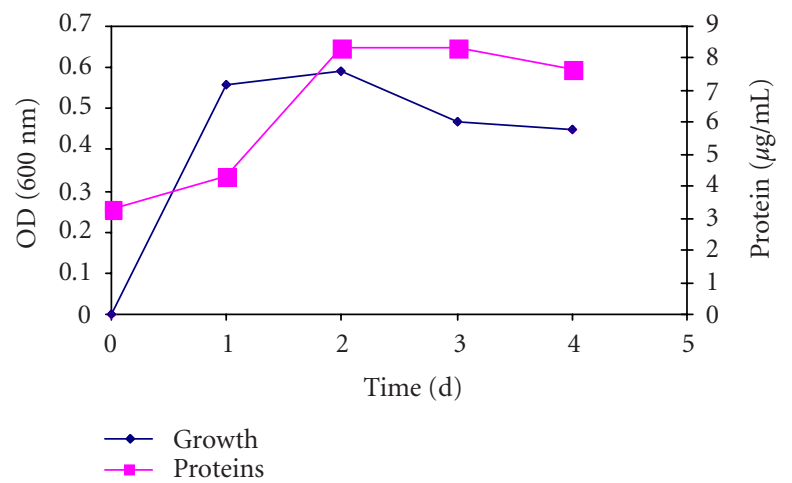

FIgURE 11. Protein production relative to growth of Pichia pastoris $\mathrm{KM} 71-\mathrm{H}^{\left(\mathrm{mut}^{\mathrm{s}}\right)}$.

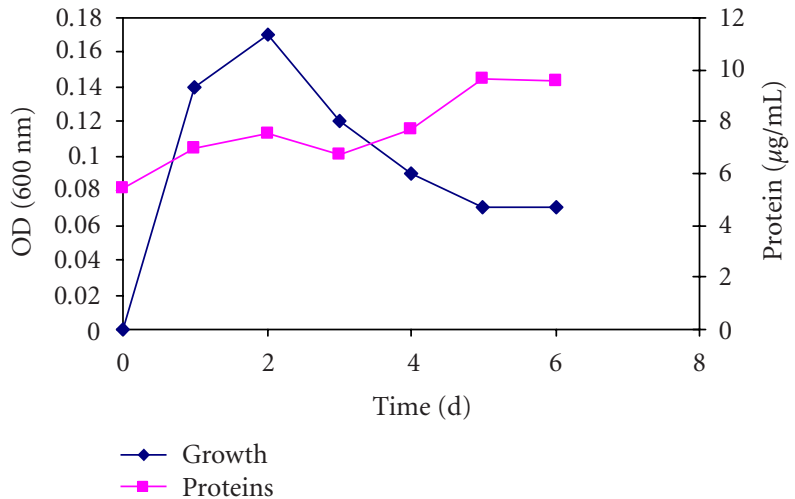

Figure 12. Protein production relative to growth of Pichia pastoris $\mathrm{X}-33^{\left(\mathrm{mut}^{+}\right)}$.

host genome. Multiple integration of foreign genes into a host genome results in increased frequency of producing high-copy number transformants, subsequently, resulting in increased expression levels of recombinant proteins (Rai and Padh [20]; Scorer et al [21]; Sreekrishna et al [22]; Yan et al [15]). 


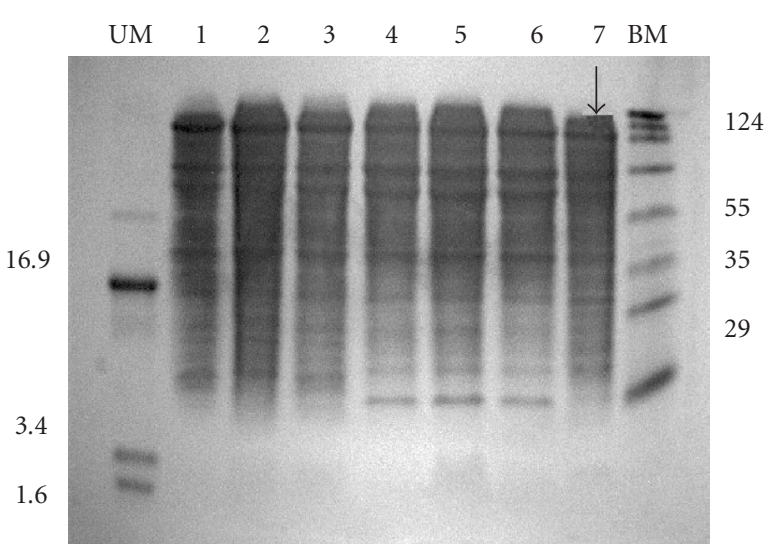

(a)

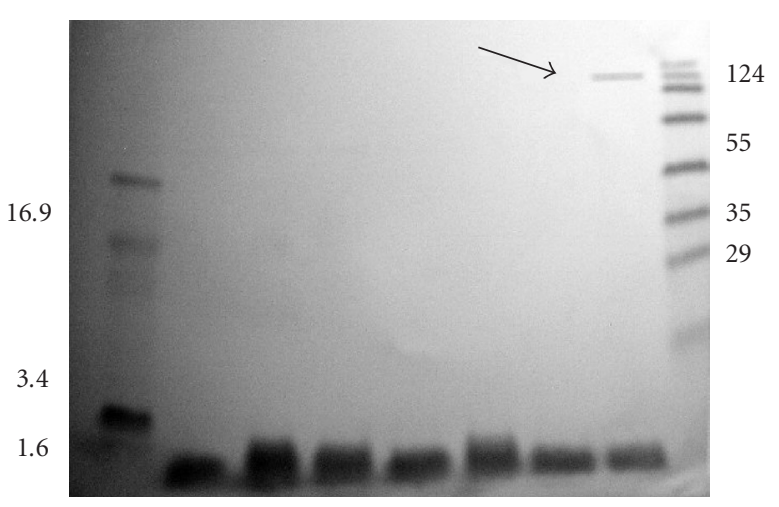

(b)

FIGURE 13. UM represents ultra-marker (peptides). BM represents broad-range marker (proteins). 1-2: $P$ pastoris X-33 induced transformants (day 3). 3: uninduced $P$ pastoris X-33 transformants (day 3). 4-5: $P$ pastoris KM71 H induced transformants (days 5 and 6). 6: uninduced $P$ pastoris KM 71H transformants (day 5). 7: P pastoris GS115/LacZ expression control. (a) SDS PAGE gel of timecourse intracellular samples of transformed $P$ pastoris $(\mathrm{KM} 71 \mathrm{H}$ and X-33) and (b) Western blot of time-course intracellular samples of transformed $P$ pastoris (KM $71 \mathrm{H}$ and $\mathrm{X}-33$ ).

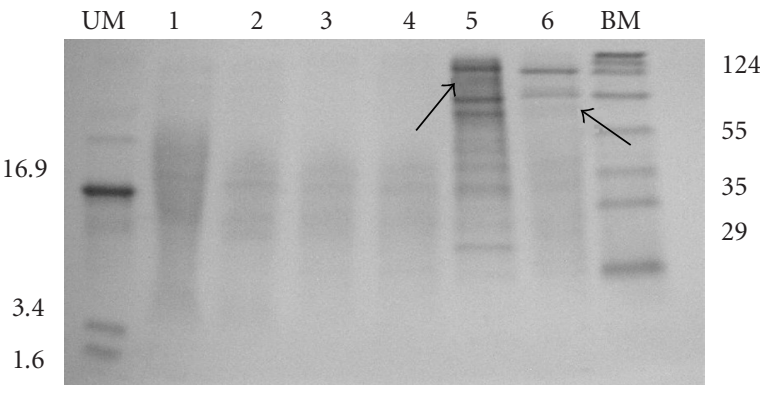

(a)

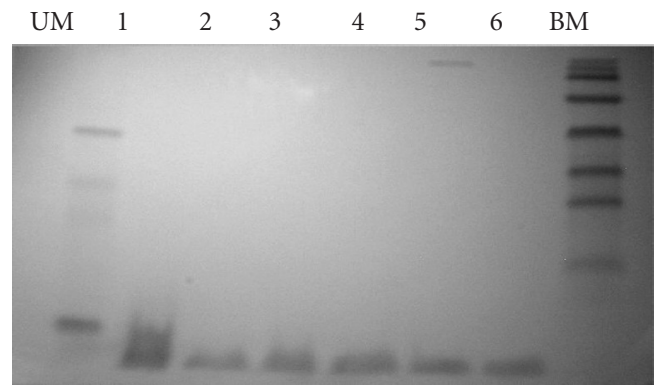

(b)

Figure 14. 1-2: Pichia pastoris X-33 clones $1 \& 2$ at day 3. 3-4: $P$ pastoris KM71H clones at days 5 \& 6. 5-6: $P$ pastoris LacZ and $P$ pastoris GS115/HSA (controls). UM represents ultra-low marker (peptides). BM represents broad-range marker (proteins). (a) SDS PAGE gel from supernatant of transformed $P$ pastoris (KM $71 \mathrm{H}$ and X-33) and (b) Western blot of supernatant from transformed $P$ pastoris (KM $71 \mathrm{H}$ and $\mathrm{X}-33)$.

\section{REFERENCES}

[1] Cole AM, Weis P, Diamond G. Isolation and characterization of pleurocidin, an antimicrobial peptide in the skin secretions of winter flounder. J Biol Chem. 1997;272(18):12008-12013.

[2] Burrowes OJ, Hadjicharalambous C, Diamond G, Lee TC. Evaluation of antimicrobial spectrum and cytotoxic activity of pleurocidin for food applications. J Food Sci. 2004;69(3):66-71.

[3] Cole AM, Darouiche RO, Legarda D, Connell N, Diamond G. Characterization of a fish antimicrobial peptide: gene expression, subcellular localization, and spectrum of activity. Antimicrob Agents Chemother. 2000;44(8):2039-2045.

[4] Jia X, Patrzykat A, Devlin RH, Ackerman PA, Iwama GK, Hancock RE. Antimicrobial peptides protect coho salmon from vibrio anguillarum infections. Appl Environ Microbiol. 2000;66(5):1928-1932.

[5] Higgins DR, Cregg JM. Methods in molecular biology. In: Pichia Protocols. New Jersey, NJ: Humana Press; 1998:1-16.

[6] d'Anjou MC, Daugulis AJ. A rational approach to improving productivity in recombinant pichia pastoris fermentation. Biotechnol Bioeng. 2001;72(1):111.

[7] Villatte F, Hussein AS, Bachmann TT, Schmid RD. Expression level of heterologous proteins in Pichia pastoris is influenced by flask design. Appl Microbiol Biotechnol. 2001;55(4):463-465.

[8] Eldin P, Pauza ME, Hieda Y, et al. High-level secretion of two antibody single chain fv fragments by Pichia pastoris. J Immunol Methods. 1997;201(1):6775. 
[9] Shen S, Sulter G, Jeffries TW, Cregg JM. A strong nitrogen source-regulated promoter for controlled expression of foreign genes in the yeast Pichia pastoris. Gene. 1998;216(1):93-102.

[10] Baumgartner P, Raemaekers RJ, Durieux A, Gatehouse A, Davies H, Taylor M. Large-scale production, purification, and characterisation of recombinant phaseolus vulgaris phytohemagglutinin E-form expressed in the methylotrophic yeast Pichia pastoris. Protein Expr Purif. 2002;26(3):394-405.

[11] Bellevik S, Summerer S, Meijer J. Overexpression of arabidopsis thaliana soluble epoxide hydrolase 1 in Pichia pastoris and characterisation of the recombinant enzyme. Protein Expr Purif. 2002;26(1):65-70.

[12] Ogawa M, Nakamura S, Atsuchi T, Tamiya T, Tsuchiya T, Nakai S. Macromolecular antimicrobial glycoprotein, achacin, expressed in a methylotrophic yeast Pichia pastoris. FEBS Lett. 1999;448(1):41-44.

[13] Reddy ST, Dahms NM. High-level expression and characterization of a secreted recombinant cationdependent mannose 6-phosphate receptor in Pichia pastoris. Protein Expr Purif. 2002;26(2):290-300.

[14] Feng W, Cai J, Pierce WM Jr, Song ZH. Expression of CB2 cannabinoid receptor in Pichia pastoris. Protein Expr Purif. 2002;26(3):496-505.

[15] Yan Y, Chen J, Li J. Overexpression of a small medicinal peptide from ginseng in the yeast Pichia pastoris. Protein Expr Purif. 2003;29(2):161-166.

[16] Koganesawa N, Aizawa T, Shimojo H, et al. Expression and purification of a small cytokine growthblocking peptide from armyworm pseudaletia separata by an optimized fermentation method using the methylotrophic yeast Pichia pastoris. Protein Expr Purif. 2002;25(3):416-425.

[17] Paramasivam M, Saravanan K, Uma K, Sharma S, Singh TP, Srinivasan A. Expression, purification, and characterization of equine lactoferrin in Pichia pastoris. Protein Expr Purif. 2002;26(1):28-34.

[18] Li H, Ma Y, Su T, Che Y, Dai C, Sun M. Expression, purification, and characterization of recombinant human neurturin secreted from the yeast Pichia pastoris. Protein Expr Purif. 2003;30(1):11-17.

[19] Butt TR, Edavettal SC, Hall JP, Mattern MR. Sumo fusion technology for difficult-to-express proteins. Protein Expr Purif. 2005;43(1):1-9.

[20] Rai M, Padh H. Expression systems for production of heterologous proteins. Current Science. 2001;80:1121-1128.

[21] Scorer CA, Clare JJ, McCombie WR, Romanos MA, Sreekrishna K. Rapid selection using G418 of high copy number transformants of Pichia pastoris for high-level foreign gene expression. Biotechnology $(\mathrm{N}$ Y). 1994;12(2):181-184.

[22] Sreekrishna K, Brankamp RG, Kropp KE, et al. Strategies for optimal synthesis and secretion of heterologous proteins in the methylotrophic yeast Pichia pastoris. Gene. 1997;190(1):55-62. 

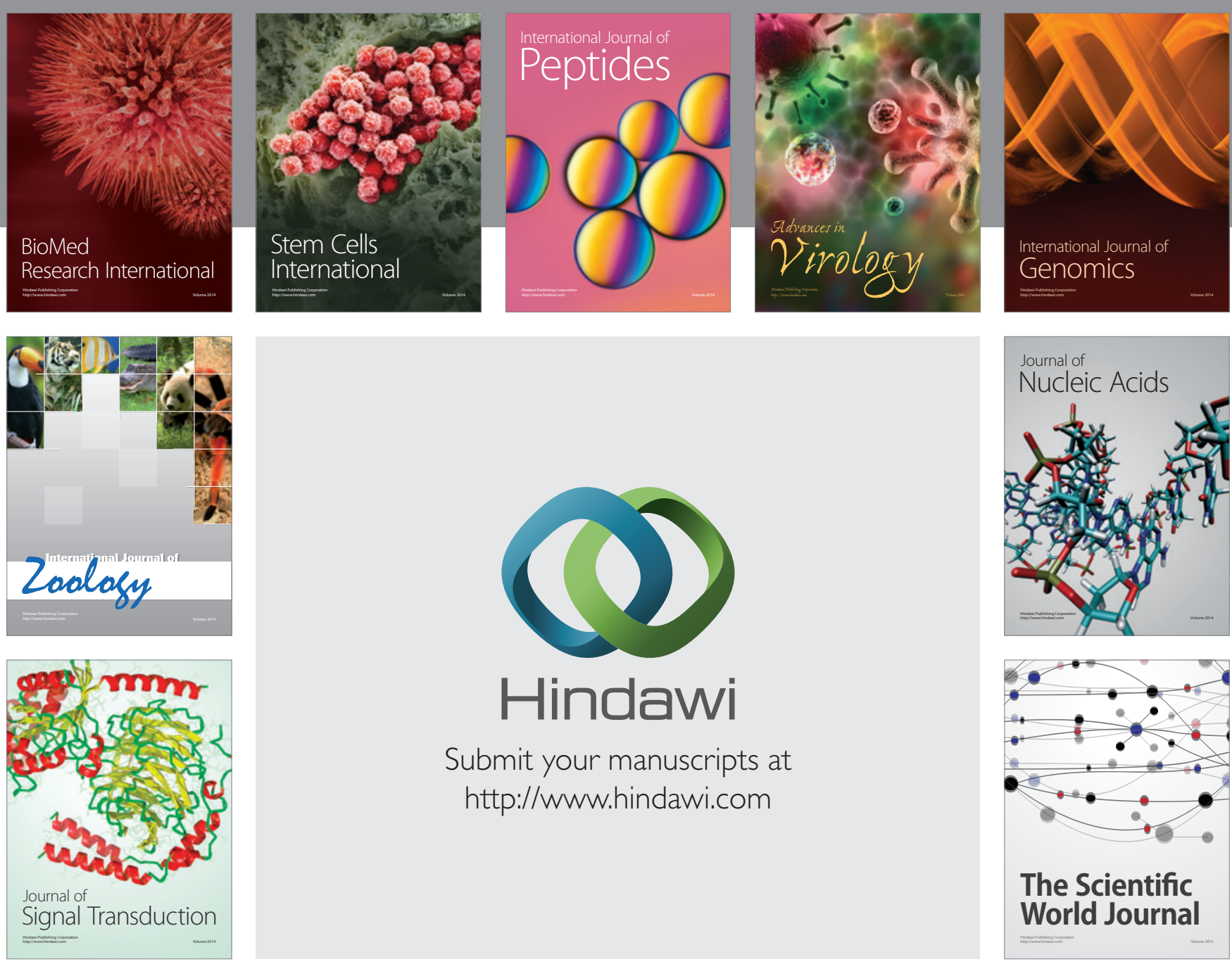

Submit your manuscripts at

http://www.hindawi.com
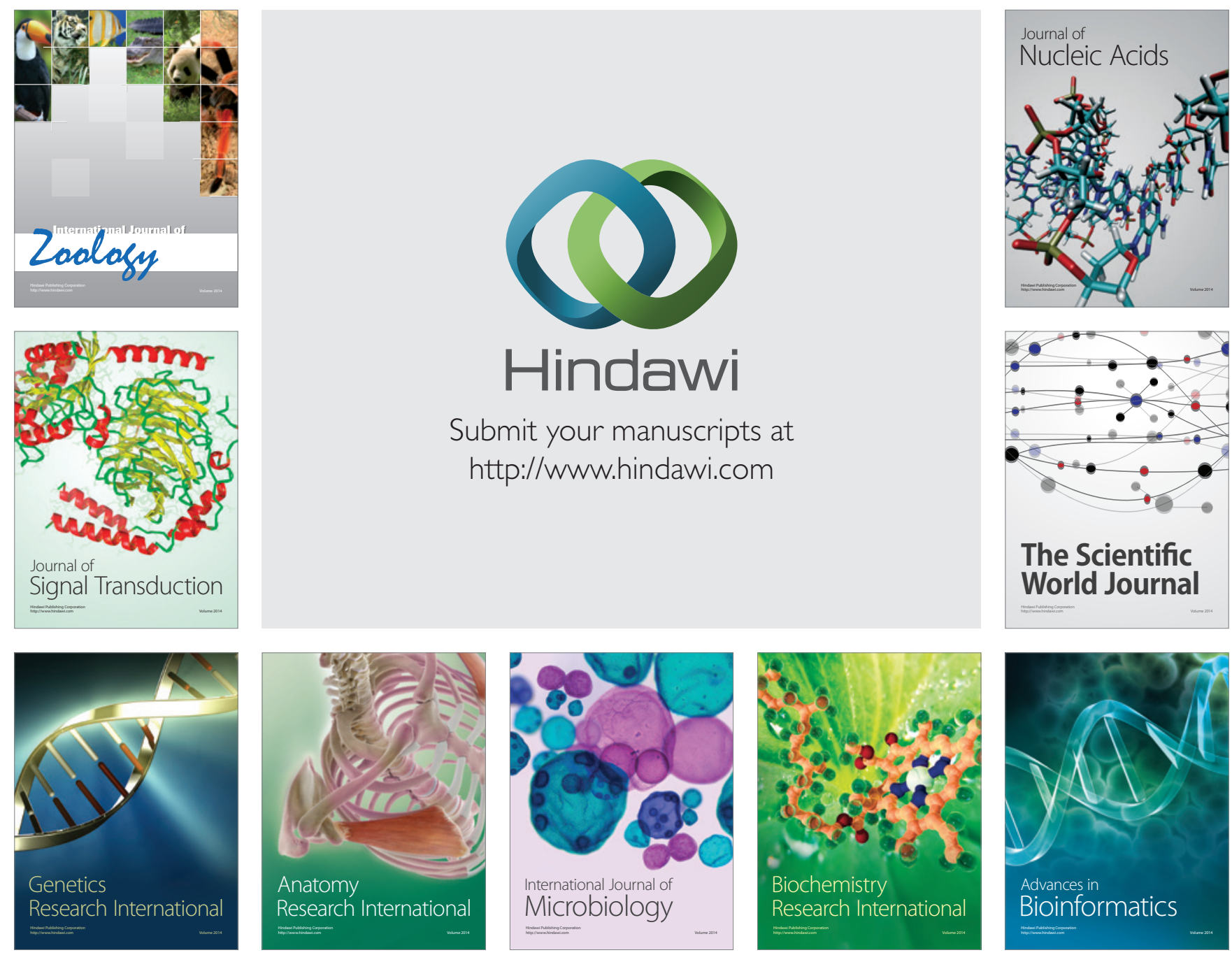

The Scientific World Journal
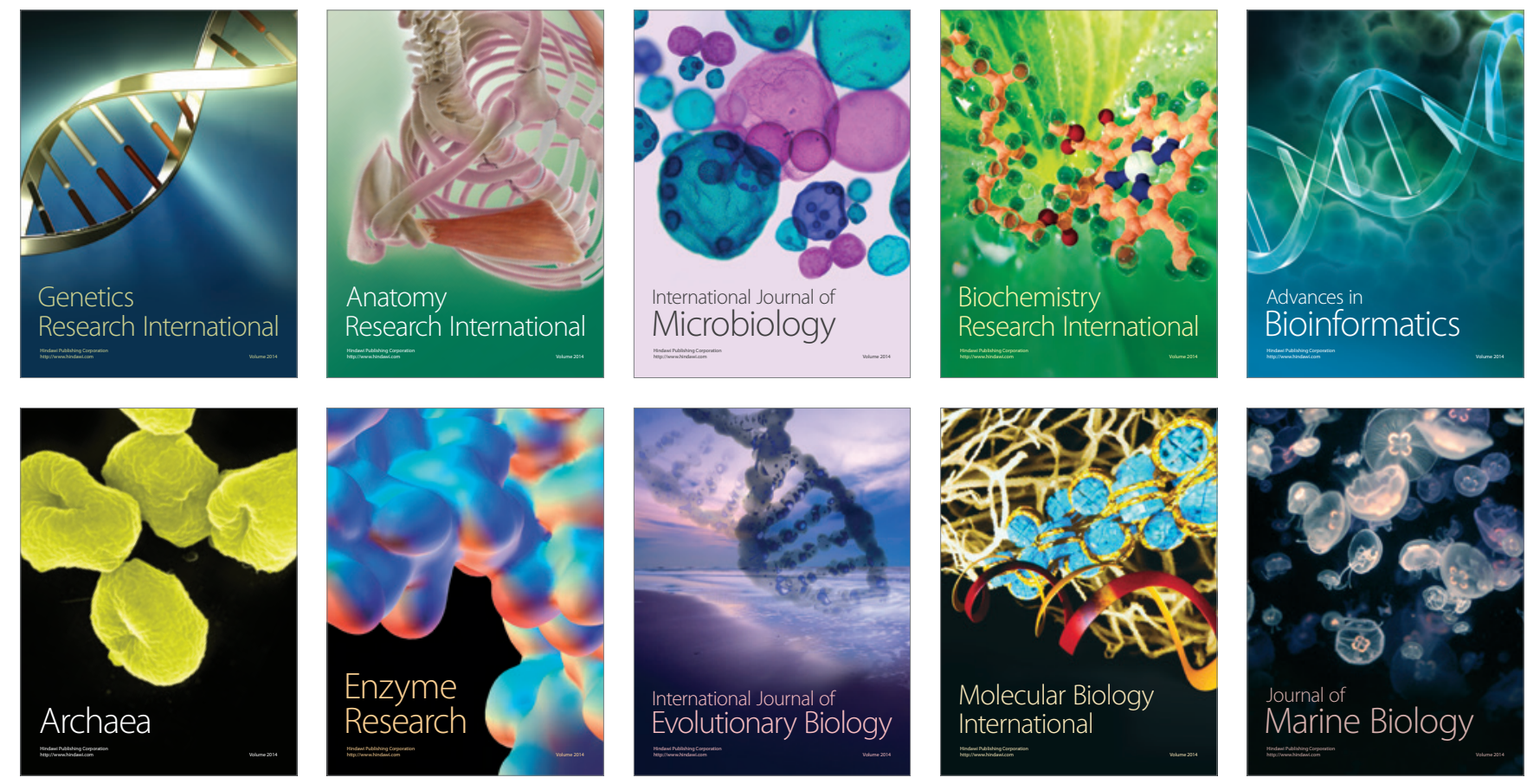